*ak RMIS View/Frint Document Cover Sheet tow

This document was retrieved from the Documentation and Records Manaqement (DRM) ISEARCH System. It is intended for Information only and may not be the most recent or updated version. Contact a Document Service Center (see Hanford Info for locations) if you need additional retrieval information.

Accession \#: D196061011

Document \#: SD-SNF-DRR-006

Title/Desc:

FINAL DESIGN REVIEW REPORT FOR K BASIN DOSE REDUCTION PROJECT

Pages: 38 


\begin{tabular}{|l|l|}
\hline $\begin{array}{l}\text { 2. To: (Receiving Organization) } \\
\text { Spent Nuclear Fuel/K Bas in } \\
\text { Projects }\end{array}$ & $\begin{array}{l}\text { 3. From: coriginating Organization) } \\
\text { Mechanisms Engineering }\end{array}$ \\
\hline $\begin{array}{l}\text { 5. Proj./Prog./Dept./Div.: } \\
\text { Dose Reduction/A.8 }\end{array}$ & $\begin{array}{l}\text { 6. Cog. Engr.: } \\
\text { L. D. Blackburn }\end{array}$ \\
\hline $\begin{array}{l}\text { 8. Originator Renarks: } \\
\text { Keywords: Spent Nuclear Fuel, K Basins, Dose Reduction, } \\
\text { Water Level }\end{array}$
\end{tabular}

Water Leve]

11. Receiver Remarks:

4. Related EDT No.

$N / A$

7. Purchase Order No.:

N/A

9. Equip./Component Ko.:

N/A

10. System/Bldg./Facility: $105 K E$

12. Major Assm. Dwg. No.: N/A

13. Permit/Permit Application No.: N/A

14. Required Response Date:

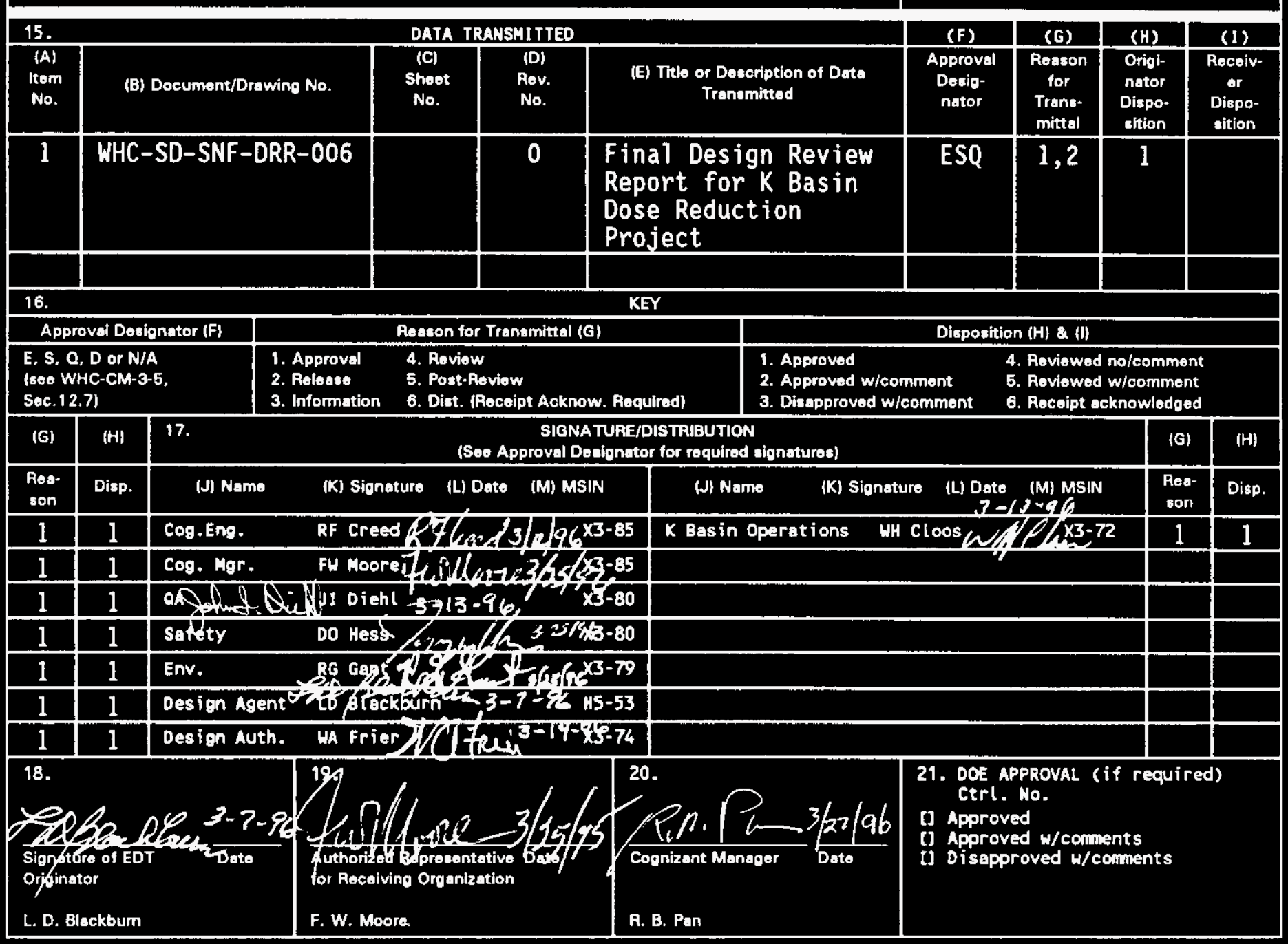

BD-7400-172-2(04/94) GEF097 


\begin{tabular}{|c|c|}
\hline BLOCK & IITLE \\
\hline$(1)^{*}$ & EOT \\
\hline (2) & To: (Receiving Organization) \\
\hline (3) & From: (Originating Organization) \\
\hline (4) & Rolated EDT No. \\
\hline$(5)^{\circ}$ & Proj./Pros./Dept./Div. \\
\hline$(6)^{*}$ & Cognizant Engineer \\
\hline (7) & Purchase Order No. \\
\hline$(8)^{*}$ & Originator Remarks \\
\hline (9) & Equipment/Component No. \\
\hline$(10)$ & Systom/BIdg./Facility \\
\hline (11) & Roceiver Remarks \\
\hline (12) & Major Assm. Dwg. No. \\
\hline (13) & Permit/Permit Application No. \\
\hline (14) & Required Response Date \\
\hline
\end{tabular}

(15). Data Transmitted
(A) * Item Number
(B) * Document/Drawing No
(C) ${ }^{*}$ Sheet No.
(D) Rev, No.
(E) Title or Description of Data Transmitted
$|F|^{*}$ Approval Designator
(G) Reoson for Transmittal
(H) Originator Disposition
(I) Receiver Disposition

(16) Key

(17) Signature/Distribution
(G) Reason
(H) Disposition
(J) Name
$(K)^{*}$ Signature
(L). Date
(M)* MSIN

(18)

Signature of EDT Originator

(19)

Authorized Representative for Receiving Organization

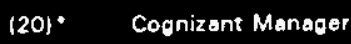

(21) DOE Approval
- Pre-aserigned EDT number.

- Enter the individual's name, title of the organization, or entity (o.e., Distribution) that the EDT is being tranemitted to.

- Enter the titlo of the org anization originating and transmitting the EDT.

- Enter EDT numbers which relate to the dato being transmitted.

- Enter the Project/Program/Department/Division titlo or Project/Program acronym or Project Number, Work Order Number or Organization Code.

- Enter the name of the individual identified as being responsible for coordinating disposition of the EDT.

- Enter related Purchase Order (P.O.) Number, if availablo

- Enter special or additional comments concerning transmittal, or "Koy" retrioval words may be entered.

- Enter equipment/component number of affected item, if appropriate.

- Enter applicable system, building or facility number, if appropriate.

c Enter special or additional comments concerning transmittal.

- Enter applicable drawing number of major assembly, if appropriate.

- Enter applicable permit or permit application number, if appropriate.

C Enter the date o response is required from individuals identified in Block 17 (Signature/Distribution).

- Enter sequential number, beginning with 1, of the information listed on EDT.

Enter the unique identification number assigned to the document or drawing being transmitted.

Enter the sheet number of the information being transmitted. If no sheet number, leavo blank.

Enter the revision number of the information being transmitted. If no revision number, loave blank.

C Enter the title of the document or drawing or a brief description of the subject if no title is identified.

A Enter the sppropriate Approval Designator (Block 15). Also, indicato the appropriate spprovale for esch item listed, i.e., SO, ESQ, otc.

- Enter the appropriate code to identify the purpose of the data transmittal (see Block 16).

- Enter the appropriate disposition code (see Block 16).

- Enter the appropriate disposition code (see Block 16).

r Number codes used in completion of Blocks 15 (G), (H), and (I), and 17 (G), (H) (Signature/Distribution).

( Enter the code of the reason for transmittal (Block 16 ).

$\ulcorner$ Enter the code for the disposition (Block 16).

- Enter the signature of the individual completing the Disposition $17(\mathrm{H})$ and the Transmittal.

n Obtain appropriate signature(s).

Enter date signature is obtained.

a Enter MSIN. Note: If Distribution Sheet is used, show entire distribution fincluding that indicated on Page 1 of the EDT) on the Distribution Sheot.

Enter the signature and date of the individual originating the EDT (entered prior to transmittal to Receiving Organization). If the EDT originator is the cognizant engineer, tign both Blocks 17 and 18

c Enter the signature and date of the individual identified by the Roceiving Organization as authorized to approve disposition of the EDT and acceptance of the data transmitted, as applicable.

- Enter the signature and date of the cognizant manager. (This signature is authorization for release.)

A Enter DOE approval lif requiredl by signature or control number that tracks the approval to a tignature, and indicate DOE action. 


\title{
FINAL DESIGN REVIEW REPORT FOR K BASIN DOSE REDUCTION PROJECT
}

\author{
L. D. Blackburn
}

ICF Kaiser Hanford Company, Richland, WA 99352

U.S. Department of Energy Contract DE-AC06-87RL10930

$\begin{array}{lll}\text { EDT/ECN: } & 615125 & \text { UC: } 510 \\ \text { Org Code: } & 57 E 00 & \text { Charge Code: } \\ \text { B\&R Code: } & \text { EW3135040 } & \text { Total Pages: } 36\end{array}$

Key Words: K Basin, Dose Reduction

Abstract: The strategy for reducing radiation dose originating from radionuclides absorbed in the $K$ East Basin concrete is to raise the pool water level to provide additional shielding. This report documents a final design review for cleaning/coating basin walls and modifying other basin components where appropriate. The conclusion of this review was that the documents developed constitute an acceptable design for the Dose Reduction Project.

TRADEMARK DISCLAIMER. Reference herein to any specific comnercial product, process, or service by trade name, trademark, manufacturer, or otherwise, does not necessarily constitute or imply its endorsenent, recommendation, or favoring by the United States Government or any agency thereof or its contractors or subcontractors.

Printed in the United States of Anerica. To obtain copies of this document, contact: MHC/BCS Docunent Control Services, P.O. Box 1970, Mailstop 16-08, Richland WA 99352, Phone (509) 372-2420; Fax (509) 376-4989.
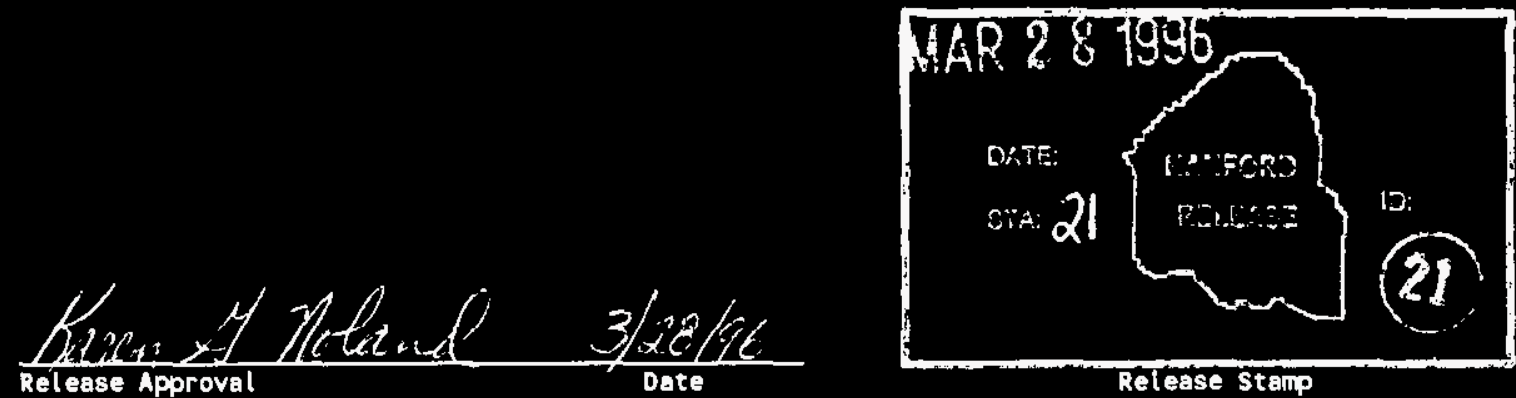

Approved for Public Release 


\section{CONTENTS}

Page

1.0 INTRODUCTION. ......................... 1

$2.0 \mathrm{SCOPE} \ldots \ldots \ldots \ldots 1$

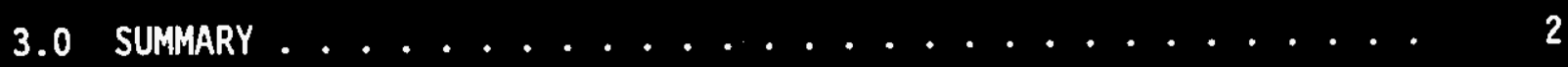

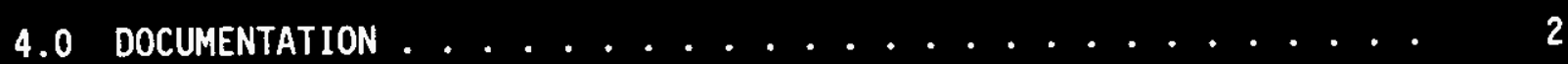
APPENDICES

APPENDIX A - List of Documents Reviewed. . . . . . . . A-1 APPENDIX B - Design Review Checklist . . . . . . . . . B-1 APPENDIX C - K Basin Dose Reduction Project Final Design Review. . . . . C C-1 APPENDIX D - Review Comment Records. . . . . . . . . . D-1 APPENDIX E - Action Item Data Base Status. . . . . . . . E E-1 APPENDIX F - Action Item List. .............. F-1 APPENDIX G - K Basin Dose Reduction Project Final Design Review. . . . G- G-1 


\section{FINAL DESIGN REVIEN REPORT FOR K BASIK DOSE REDUCTION PROJECT}

\subsection{INTRODUCTION}

A major source of radiation above the water level in the $105 \mathrm{~K}$-East Bas in is the radionuclide inventory absorbed into the concrete walls. The current strategy for reducing radiation dose is to raise the water level in the basin. The concrete above the present water level must be coated with a material that will prevent absorption of radionuclides into the newly submerged portion of the wall. Early work on dose reduction identified design concepts that did not involve raising the water level. Project plans and documents were based on removal of significant thickness of concrete both above and below the water line, followed by application of coating to prevent reabsorption of radionuclides. Only recently have all the issues related to raising the water level been resolved in favor of that strategy. This approach for dose reduction was reported in Preliminary Design Review Report for $K$ Basin Dose Reduction Project, WHC-SD-SNF-DRR-004 Rev. 0. The fina1 design review reported here was conducted to ensure that project design documentation was complete and adequate.

\subsection{SCOPE}

The documents reviewed are identified in the List of Documents appended to this report. Copies of the documents are retained in $K$ Basin Project files.

This review covered the cleaning and coating of the basin walls above the present water level, and the design of an extension of the cartridge filter pit wall to prevent overflow of basin water into the pit. Design of equipment for the clean and coat task was addressed in separate reviews conducted in parallel with the present one. Results of those reviews are documented in Conceptual Design Review Report for $K$ Basin Dose Reduction Project Clean and Coat Task, WHC-SD-SNF-DRR-003, Rev. 0 and in Final Design Review Report for $K$ Basin Dose Reduction Project Clean and Coat Task, WHC-SD-SNF-DRR-005, Rev. 0 . Activities covered in those reports are not included here. Modifications to skimmer weirs and weasel pit isolation screen are necessary before raising the water level, but design of these modifications is not within the scope of this review. 


\subsection{SUNMARY}

The design review process included individual review of documentation by committee members followed by a Design Review Meeting held on February 22,1996. At the Design Review Meeting, all comments submitted by committee members and dispositions proposed by the design team were reviewed and discussed, as necessary. The action items from the Preliminary Design Review were reviewed and completed items were identified as closed. A design review checklist was completed. Two checklist questions answered "No" are covered by action items. Checklist questions $A .9$ and $C .3$ will be addressed in Items 51 and 68, respectively, of the Action Item Data Base (Appendix E). The conclusion of the meeting was that, subject to completion of items identified in either the action item list provided herein or the action item data base, the design documentation is fully acceptable for Dose Reduction Project activities.

\subsection{DOCUNENTATION}

The documentation provided as part of this report includes:

List of Documents that were reviewed (Appendix A);

Copy of completed Design Review Checklist (Appendix B);

List of Design Review committee members (Appendix C);

Copies of Review Comment Records generated by committee members (Appendix D);

Status of Preliminary Design Review comments in the Action Item Data Base (AIDB) (Appendix E).

List of Action Items that should be addressed in completing design documentation (Appendix F);

Meeting minutes for Design Review Meeting (Appendix G). 


\section{APPENDIX A}

\section{LIST OF DOCUMENTS REVIENED}

Numbered Documents

ECN 190567 to and original of Functions and Requirements for the $105 \mathrm{~K}$-East Basin Dose Reduction Project, WHC-SD-SNF-FRD-001, Rev. 0.

Project Management Plan for Project A.8, Loadout Dose Management, WHC-SD-SNF-PMP-007, Rev. 0.

Design Review Report for the $105 \mathrm{~K}$-East Basin Dose Reduction Concept, WHC-SD-SNF-DRR-002, Vo1. 1, Rev. 0.

Conceptual Design Review Report for $K$ Basin Dose Reduction Project Clean and Coat Equipment, HHC-SD-SNF-DRR-003.

Preliminary Design Review Report for $K$ Basin Dose Reduction Project, WHC-SD-SNF-DRR-004.

Dose Reduction Cleaning and Coating Basin Walls, Waste Management Plan WMP-01-96.

Dose Reduction Extension of Tech View Pit Wall, Waste Management Plan WMP-02-96.

ECN 190566, direct revision of Specification for $105 K$ East Basin Dose Reduction Project Decon and Seal Task, WHC-S-0375, Rev. 0.

ECN 190564 to Drawing H-1-34692, Rev. 1, Sh. 1. (Coating Installation)

ECN 611951 to Drawing H-1-34710, Rev. 1, Sh. 1. (Cartridge Filter Pit Dam Installation) and ALARA Review Meeting Minutes.

WHC Internal Memo 01880-95-104, Categorically Excluded Cleaning and Coating of K-East Bas in Walls, $100 \mathrm{~K}$ Area, Hanford Site, Richland, Washington (author: R. H. Engelmann, NEPA Services).

WHC Internal Memo 8M730-RLS-95-014, KE-Basin Dose Reduction by Cleaning, Coating, and Raising the Water Level (author: R. L. Simons, Nuclear Physics and Shielding).

Modification 1 of Purchase Order MDK-XVC-406988 and Revision 1 of Statement of Work.

Skimmer Weir Design Drawing H-1-34741, Sheets 2 and 3 (Unreleased). 


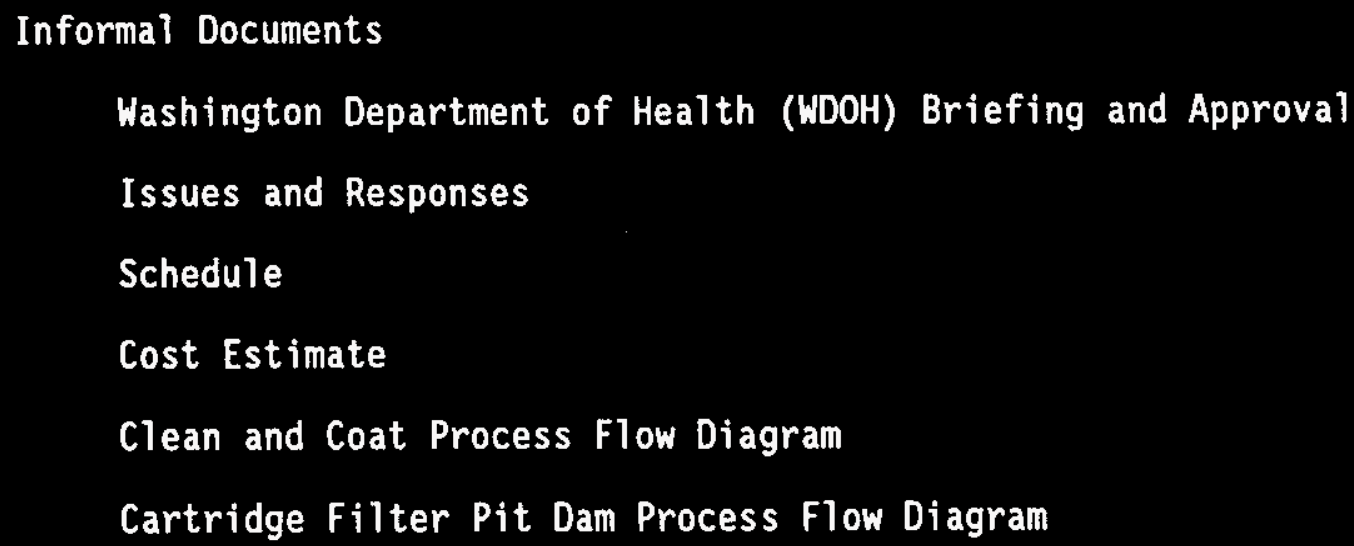


In answering the checklist questions, a "No" answer should require the preparation of a written comment per the Design Review guidelines.

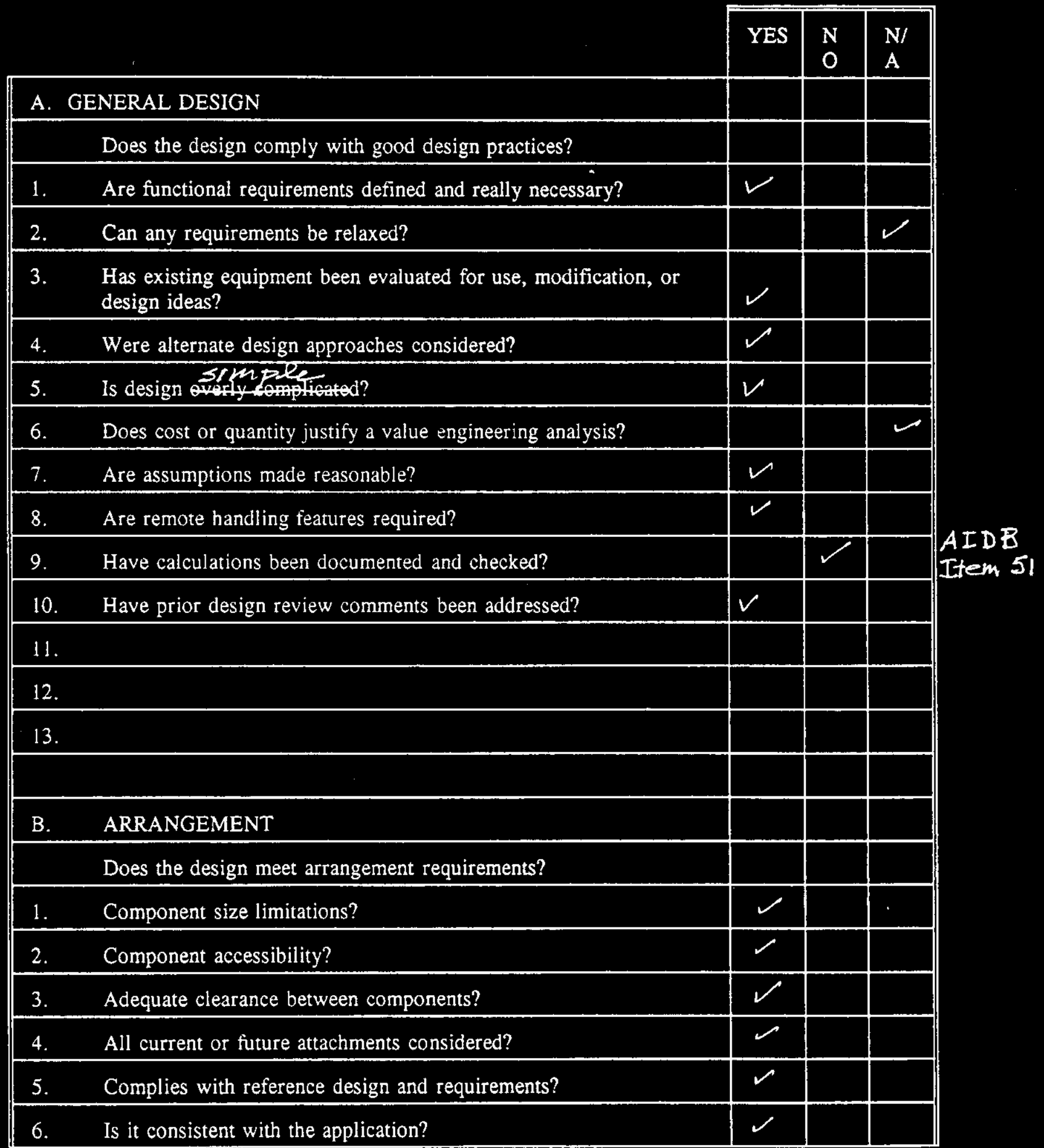

D: \SNF_ES \DOSE_RED \CLN_COAT \PROCURMT \APPEND-D.WPD August 28, 1995 


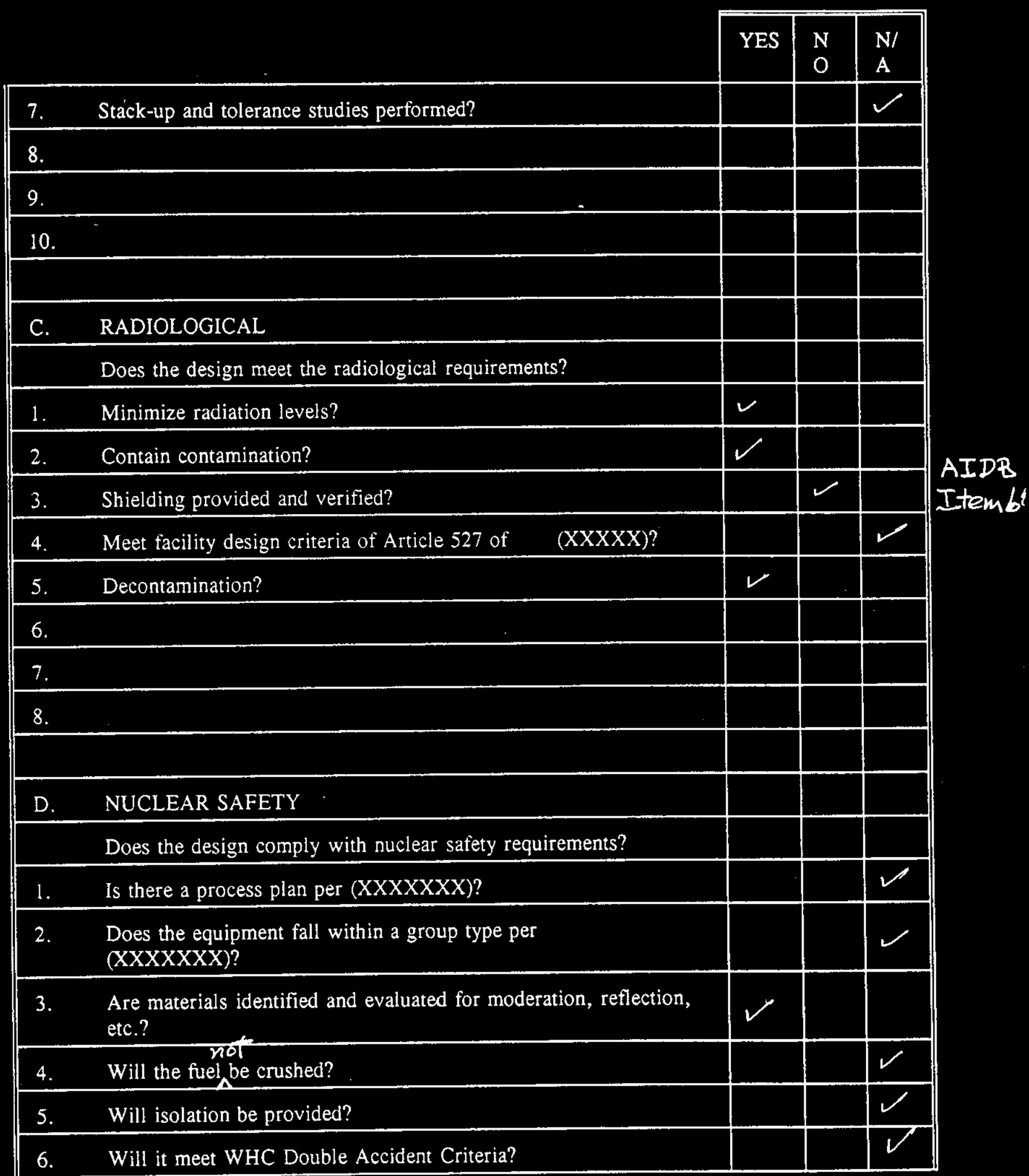

D: \SNF_ES\DOSE_RED \CLN_COAT \PROCURMT \APPEND-D.WPD August 28, 1995 


\begin{tabular}{|c|c|c|c|c|}
\hline & & YES & $\begin{array}{l}\mathbf{N} \\
\mathbf{O} \\
\end{array}$ & $\begin{array}{l}\mathrm{N} / \\
\mathrm{A}\end{array}$ \\
\hline \multicolumn{5}{|l|}{7.} \\
\hline \multicolumn{5}{|l|}{8.} \\
\hline \multicolumn{5}{|l|}{9.} \\
\hline \multicolumn{5}{|c|}{ E. OPERATION } \\
\hline & Does the design comply with operational needs and limitations? & & & \\
\hline 1. & Reliability provisions? & $\checkmark$ & & \\
\hline 2. & Utilized preferred or commonly used components? & $\checkmark$ & & \\
\hline 3. & Provide fabrication and as-built drawings? & $\checkmark$ & & \\
\hline 4. & Provide operating instructions? & & & $\checkmark$ \\
\hline 5. & Safety provisions? & $r$ & & \\
\hline 6. & Meet intended use and foreseeable misuse? & $\checkmark$ & & \\
\hline 7. & Provide interlocks/safety devices? & & & 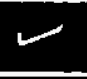 \\
\hline 8. & Operation and maintenance provisions? & $\checkmark$ & & \\
\hline 9. & Simple operations? & $\checkmark$ & & \\
\hline 10. & Understandable and operable controls? & & & $\checkmark$ \\
\hline & Spare parts availability? & & & $\checkmark$ \\
\hline 12. & Environment effects of: & & & \\
\hline a. & Extremes of service voltages? & & & $\nu$ \\
\hline & Extremes of service water hardness? & & & $\swarrow$ \\
\hline c. & Extremes of service pressure? & & & $\checkmark$ \\
\hline d. & Extremes of service temperature? & & & $\checkmark$ \\
\hline e. & Extremes of external vibration? & & & $\checkmark$ \\
\hline f. & Extremes of external shock? & & & $\checkmark$ \\
\hline g. & Extremes of ambient temperature? & $v$ & & \\
\hline
\end{tabular}




\section{DESIGN REVIEW CHECKLIST}

\begin{tabular}{|c|c|c|c|c|}
\hline & & \\
\hline & & YES & $\begin{array}{l}\mathrm{N} \\
\mathrm{O} \\
\end{array}$ & $\begin{array}{l}\mathrm{N} / \\
\mathrm{A}\end{array}$ \\
\hline h. & Extremes of humidity? & & & $\checkmark$ \\
\hline i. & Foreign material (sand, grit, oil, lint, dirt, etc.)? & & & $\checkmark$ \\
\hline j. & Corrosive ambients (water, acids, etc.)? & & & $\checkmark$ \\
\hline k. & Extremes of external magnetic fields? & & & $\checkmark$ \\
\hline 1. & Weather extremes? & & & $\checkmark$ \\
\hline $\mathrm{m}$. & Radio interference? & & & $\checkmark$ \\
\hline $\mathrm{n}$. & Nuclear radiation? & & & $\checkmark$ \\
\hline 0. & Supplementary products (detergents, bleaches, oils, grease, etc.)? & & & $\checkmark$ \\
\hline 13. & Effect on environment of: & & & \\
\hline a. & Appearance? & & & $\checkmark$ \\
\hline b. & Noise? & & & $\checkmark$ \\
\hline c. & Odor? & & & $V$ \\
\hline d. & Temperature? & & & $\swarrow$ \\
\hline e. & Vibration? & & & $\Upsilon$ \\
\hline $\mathrm{t}$ & Light? & & & $\checkmark$ \\
\hline g. & Radiation? & & & レ \\
\hline h. & Exhaust? & & & $\checkmark$ \\
\hline i. & Space? & & & $\checkmark$ \\
\hline $\mathrm{j}$. & Packaging disposal/recycle? & & & $\swarrow$ \\
\hline $\mathrm{k}$. & Final product disposal/recycle? & & & $\nu$ \\
\hline & 1. Material content? & & & \\
\hline & 2. Hazardous materials? & & & \\
\hline & 3. Lubricants? & & & \\
\hline 14. & \multirow{2}{*}{ Human engineering factors? } & & & $\checkmark$ \\
\hline 15. & & & & \\
\hline
\end{tabular}

D: \SNF_ES\DOSE_RED \CLN_COAT\PROCURMT \APPEND-D.WPD August 28, 1995 


\begin{tabular}{|c|c|c|c|c|}
\hline & & \\
\hline & & YES & $\begin{array}{l}N \\
0 \\
\end{array}$ & $\begin{array}{l}\mathrm{N} / \\
\mathrm{A}\end{array}$ \\
\hline \multicolumn{5}{|l|}{16.} \\
\hline \multicolumn{5}{|l|}{17.} \\
\hline \multicolumn{5}{|l|}{18.} \\
\hline \multirow[t]{2}{*}{ F. } & FAILURE & & & \\
\hline & Does the design account for failure modes? & & & \\
\hline 1. & Failure modes identified? & $\checkmark$ & & \\
\hline 2. & How failures detected? & & & $\checkmark$ \\
\hline 3. & Compensating factors for failures? & & & 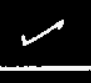 \\
\hline 4. & Recovery from failures? & & & $\checkmark$ \\
\hline 5. & Repairable? & & & $\checkmark$ \\
\hline 6. & Is a Failure Mode and Effect Analysis needed? & & & 7 \\
\hline \multicolumn{5}{|c|}{ (a) } \\
\hline \multicolumn{5}{|l|}{8.} \\
\hline \multicolumn{5}{|l|}{9.} \\
\hline \multirow[t]{2}{*}{ G. } & RELIABILITY & & & \\
\hline & Does the design meet reliability requirements? & & & \\
\hline 1. & $\begin{array}{l}\text { Maximum stresses within limits through full range of travel, load, } \\
\text { voltage, etc.? }\end{array}$ & $\checkmark$ & & \\
\hline 2. & Derating utilized? & & & 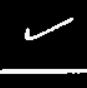 \\
\hline 3. & Simplicity optimized? & $\checkmark$ & & \\
\hline 4. & Failure modes of critical elements analyzed? & 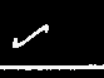 & & \\
\hline & Optimum use of standard proven párts? & $\checkmark$ & & \\
\hline 6. & Correct current problems and complaints? & $\checkmark$ & & \\
\hline
\end{tabular}

$$
D: \backslash S N F \_E S \backslash D O S E \_R E D \backslash C L N \text { CCOAT } \backslash \text { PROCURMT \APPEND-D.WPD August 28, } 1995
$$


Rev. 0

DESIGN REVIEW CHECKLIST

\begin{tabular}{|c|c|c|c|c|}
\hline & & \\
\hline & & YES & $\begin{array}{l}\mathrm{N} \\
\mathrm{O}\end{array}$ & $\begin{array}{l}\text { N/ } \\
\mathrm{A}\end{array}$ \\
\hline 7. & Similar design data considered? & $\checkmark$ & & \\
\hline 8. & Allowable stresses per $(\mathrm{XXXXX)}$ ? & $\bar{v}$ & & \\
\hline \multicolumn{5}{|l|}{9.} \\
\hline \multicolumn{5}{|c|}{10.} \\
\hline \multicolumn{5}{|c|}{11.} \\
\hline \multirow[t]{2}{*}{$\mathrm{H}$. } & MATERIALS & & & \\
\hline & Does the design meet materials requirements? & & & \\
\hline 1. & Allowable materials for specific function and (XXXXX)? & $\checkmark$ & & \\
\hline 2. & Prohibited materials per (XXXXX)? & & & $\checkmark$ \\
\hline 3. & Corrosion resistance? & $\checkmark$ & & \\
\hline 4. & Cleanliness requirements? & 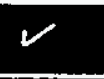 & & \\
\hline 5. & Galling? & & & $\checkmark$ \\
\hline 6. & Surface wear? & & & $\checkmark$ \\
\hline 7. & Electrical/bearing/lubricants, etc. per $(\mathrm{XXXXX)}$ ? & & & $\longleftarrow$ \\
\hline \multicolumn{5}{|l|}{8.} \\
\hline \multicolumn{5}{|l|}{9.} \\
\hline \multicolumn{5}{|c|}{10.} \\
\hline \multirow[t]{2}{*}{ I. } & INTERFACES & & & \\
\hline & Does the design comply with facility interface requirements? & & & \\
\hline 1. & Will it fit through doors and openings? & 1 & & \\
\hline 2. & Will it require support equipment and utilities? & $\checkmark$ & & \\
\hline 3. & Is there adequate visibility of operation? & $\checkmark$ & & \\
\hline 4. & Is there operation access? & $\checkmark$ & & \\
\hline
\end{tabular}

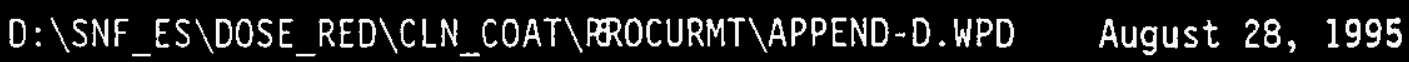




\begin{tabular}{|c|c|c|c|c|}
\hline & & \\
\hline & & YES & $\begin{array}{l}\mathrm{N} \\
\mathrm{O}\end{array}$ & N/ \\
\hline 5. & Protection for fire, water space? & & & $\checkmark$ \\
\hline \multicolumn{5}{|l|}{6.} \\
\hline \multicolumn{5}{|l|}{7.} \\
\hline \multicolumn{5}{|l|}{8.} \\
\hline \multirow[t]{2}{*}{ J. } & MANUFACTURING & & & \\
\hline & Does the design minimize manufacturing problems by: & & & \\
\hline 1. & $\begin{array}{l}\text { Utilizing existing equipment for fabrication, finishing, assembly, } \\
\text { calibration, testing, packaging, etc.? }\end{array}$ & $\checkmark$ & & \\
\hline 2. & Utilizing more efficient new processes, equipment, and facilities? & $\checkmark$ & & \\
\hline 3. & Avoiding hazardous operations? & $\nu$ & & \\
\hline 4. & Optimizing tool/piece costs? & & & $\checkmark$ \\
\hline 5. & Permitting maximum standardization? & & & $\checkmark$ \\
\hline 6. & $\begin{array}{l}\text { Specifying tolerances consistent with acceptable processes and } \\
\text { equipment? }\end{array}$ & $\checkmark$ & & \\
\hline 7. & $\begin{array}{l}\text { Correcting or avoiding previous or current manufacturing } \\
\text { problems? }\end{array}$ & & & $r$ \\
\hline 8. & Minimizing operations requiring special skills or special attention? & & & $\checkmark$ \\
\hline 9. & $\begin{array}{l}\text { Clearly identifying critical parameters to be controlled during the } \\
\text { procurement and manufacturing cycle? }\end{array}$ & & & $\checkmark$ \\
\hline 10. & Specifying materials conforming to standards? & $\checkmark$ & & \\
\hline 11. & Being clearly and completely described on drawings? & $\checkmark$ & & \\
\hline \multicolumn{5}{|l|}{12.} \\
\hline \multicolumn{5}{|l|}{13.} \\
\hline \multicolumn{5}{|l|}{14.} \\
\hline $\mathrm{K}$. & QUALITY ASSURANCE & & & \\
\hline
\end{tabular}




\begin{tabular}{|c|c|c|c|c|}
\hline & & \\
\hline & & YES & $\begin{array}{l}\mathbf{N} \\
\mathrm{O}\end{array}$ & $\begin{array}{l}\mathrm{N} / \\
\mathrm{A}\end{array}$ \\
\hline & Does the design provide for testing and quality assurance? & & & \\
\hline 1. & Inspection and testing of fabrication? & $\checkmark$ & & \\
\hline 2. & Functional tests? & & & $\sim$ \\
\hline 3. & Load tests? & & & $\checkmark$ \\
\hline 4. & Special tests? & & & $\checkmark$ \\
\hline 5. & Supplier test specification? & & & $\swarrow$ \\
\hline 6. & Test documentation? & & & $r$ \\
\hline 7. & Acceptance criteria? & $\checkmark$ & & \\
\hline 8. & Westinghouse inspection? & & & $\checkmark$ \\
\hline 9. & Post installation tests? & & & $\checkmark$ \\
\hline 9. & Post installation tests? & & & $\swarrow$ \\
\hline 10. & Records and certifications? & $\checkmark$ & & \\
\hline \multicolumn{5}{|c|}{11.} \\
\hline \multicolumn{5}{|c|}{12.} \\
\hline \multicolumn{5}{|c|}{13.} \\
\hline \multirow[t]{2}{*}{ L. } & INSTALLATION & & & \\
\hline & Does the design consider installation requirements? & & & \\
\hline 1. & Is weight and size compatible with lifting equipment? & 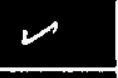 & & \\
\hline 2. & Are there lifting and handling aids? & $\checkmark$ & & \\
\hline 3. & Center-of-gravity identified? & 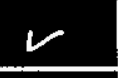 & & \\
\hline 4. & Vendor responsibilities defined? & & & $\checkmark$ \\
\hline \multicolumn{5}{|l|}{5.} \\
\hline \multicolumn{5}{|l|}{6.} \\
\hline 7. & & & & \\
\hline
\end{tabular}




\section{DESIGN REVIEW CHECKLIST}

\begin{tabular}{|c|c|c|c|c|}
\hline & & \\
\hline & & YES & $\begin{array}{l}N \\
0\end{array}$ & $\begin{array}{l}\mathrm{N} / \\
\mathrm{A}\end{array}$ \\
\hline \multirow[t]{2}{*}{ M. } & PACKAGING & & & \\
\hline & $\begin{array}{l}\text { Does the design minimize packaging, shipping, handling, and } \\
\text { warehousing problems by: }\end{array}$ & & & \\
\hline 1. & Utilizing standard packaging? & & & 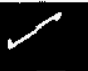 \\
\hline 2. & $\begin{array}{l}\text { Minimizing offsets, projections, etc. which require additional } \\
\text { packing material? }\end{array}$ & & & $\swarrow$ \\
\hline 3. & Withstanding applicable transportation tests? & & & $\checkmark$ \\
\hline 4. & Considering shipping and warehousing environment? & & & $\Upsilon$ \\
\hline a. & Shock? & & & $\swarrow$ \\
\hline b. & Vibration? & & & $\checkmark$ \\
\hline c. & Temperature extremes? & & & $\swarrow$ \\
\hline d. & Humidity extremes? & & & $\checkmark$ \\
\hline e. & Handling equipment? & & & $\longleftarrow$ \\
\hline f. & Sand and dust? & & & $\checkmark$ \\
\hline 5. & $\begin{array}{l}\text { Permitting shape and size of packaged product which would } \\
\text { optimize rail car and truck loading? }\end{array}$ & & & $\checkmark$ \\
\hline 6. & Providing clear handling instructions on outside of packaging? & & & $\checkmark$ \\
\hline 7. & Considering disposal of used packaging material? & & & $\checkmark$ \\
\hline \multicolumn{5}{|l|}{8.} \\
\hline \multicolumn{5}{|l|}{9.} \\
\hline \multicolumn{5}{|l|}{10.} \\
\hline \multirow[t]{2}{*}{ N. } & LAWS AND SPECIFICATIONS & & & \\
\hline & $\begin{array}{l}\text { Does the design comply with applicable laws and agency } \\
\text { requirements? }\end{array}$ & & & \\
\hline
\end{tabular}


DESIGN REVIEH CHECKLIST

\begin{tabular}{|c|c|c|c|c|}
\hline & & \\
\hline & & YES & $\begin{array}{l}\mathrm{N} \\
\mathrm{O}\end{array}$ & $\begin{array}{l}\mathrm{N} / \\
\mathrm{A}\end{array}$ \\
\hline 1. & $\begin{array}{l}\text { Does the product comply with applicable laws and agency } \\
\text { requirements? }\end{array}$ & & & \\
\hline a. & State laws? & 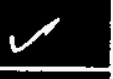 & & \\
\hline b. & Federal laws? & $\checkmark$ & & \\
\hline 2. & $\begin{array}{l}\text { Does the product comply with applicable agency standards or } \\
\text { guidelines? }\end{array}$ & & & \\
\hline a. & Underwriters' lab? & & & $\checkmark$ \\
\hline b. & OSHA? & 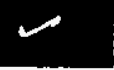 & & \\
\hline c. & ANSI? & 2 & & \\
\hline d. & DOE? & $\checkmark$ & & \\
\hline e. & NEMA? & & & 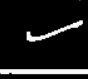 \\
\hline f. & NEPA? & $\checkmark$ & & \\
\hline g. & DOH? & $\checkmark$ & & \\
\hline \multicolumn{5}{|l|}{ h. } \\
\hline \multicolumn{5}{|l|}{ i. } \\
\hline 3. & Local requirements? & & & \\
\hline a. & Radiological Controls? & $\checkmark$ & & \\
\hline b. & Nuclear Safety? & & & $\checkmark$ \\
\hline c. & Lifting and Handling? & $\checkmark$ & & \\
\hline d. & Safety? & $\checkmark$ & & \\
\hline e. & Fire Protection? & & & $\checkmark$ \\
\hline \multicolumn{5}{|l|}{ f. } \\
\hline \multicolumn{5}{|l|}{ g. } \\
\hline \multicolumn{5}{|l|}{ h. } \\
\hline i. & & & & \\
\hline
\end{tabular}




\section{APPENDIX C}

\section{$K$ BASIN DOSE REDUCTION PROJECT}

FINAL DESIGN REVIEN

\section{CONHITTEE WENBERS}

L. D. B1 ackburn

L. E. Formo

W. H. Cloos

J. I. Dieh1

W. A. Frier

R. G. Gant

D. 0 . Hess

W. J. Millsap
Chairman

ICF-KH Construction Forces

Operations

Operations Quality Assurance

Engineering (Design Authority)

Environmental

Operational Safety

ALARA 
WHC-SD-SNF-DRR-006

Rev. 0

APPENDIX D

Review Comment Records 


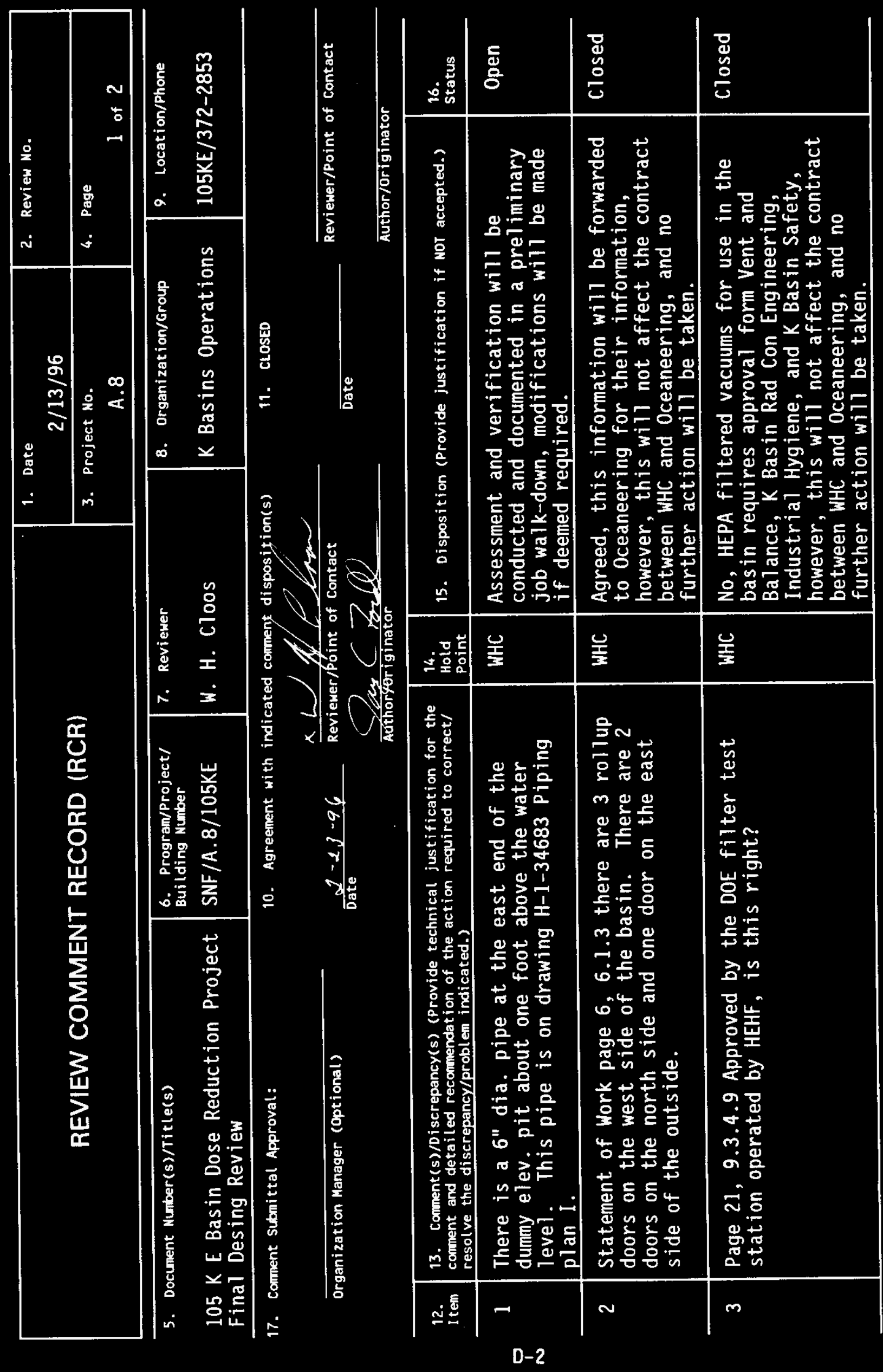

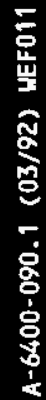




\begin{tabular}{|c|c|c|c|}
\hline & $\sim$ & 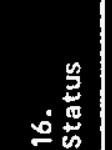 & 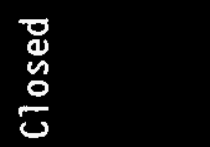 \\
\hline 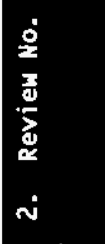 & 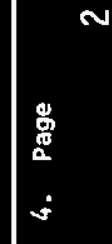 & 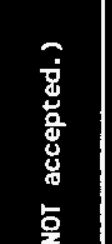 & 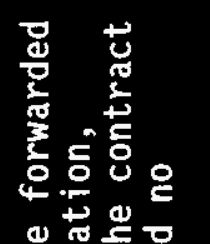 \\
\hline 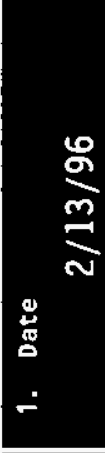 & $\mid \begin{array}{ll} & \\
\dot{0} & \dot{\alpha} \\
\frac{\mathrm{z}}{\mathrm{d}} & \\
\frac{\mathrm{o}}{\mathrm{o}} & \\
\frac{\mathrm{L}}{\alpha} & \\
\dot{m} & \end{array}$ & 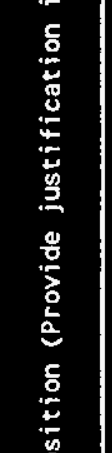 & 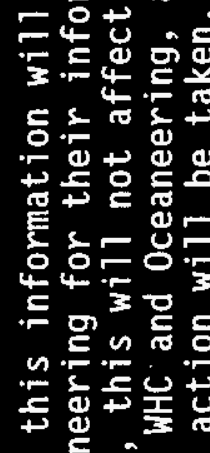 \\
\hline \multirow{4}{*}{\multicolumn{2}{|c|}{ 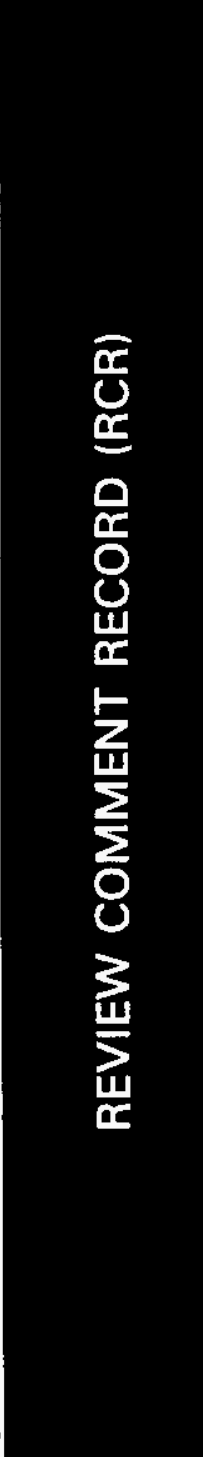 }} & $\begin{array}{l}\stackrel{\infty}{a} \\
\dot{n} \\
\dot{n}\end{array}$ & 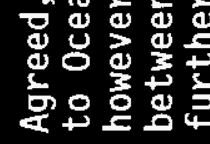 \\
\hline & & 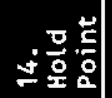 & $\frac{\varphi}{\frac{1}{z}}$ \\
\hline & & 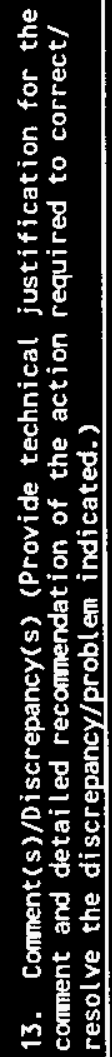 & 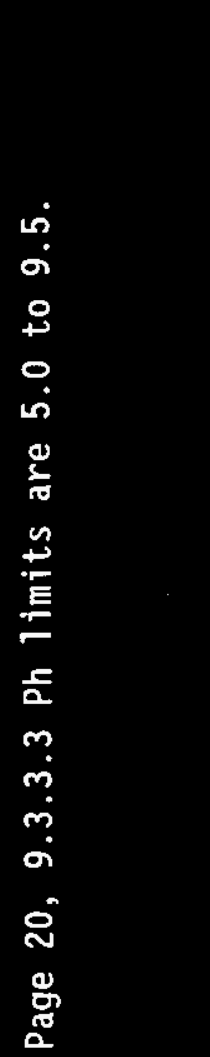 \\
\hline & & 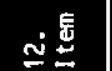 & + \\
\hline
\end{tabular}




\begin{tabular}{|c|c|c|}
\hline \multirow{2}{*}{ REVIEW COMMENT RECORD (RCR) } & $\begin{array}{l}\text { 1. Date } \\
22 / 20 / 9\end{array}$ & 2. Review No. \\
\hline & $\begin{array}{r}\text { 3. Project No. } \\
\text { A. } 8\end{array}$ & 4. Page \\
\hline
\end{tabular}

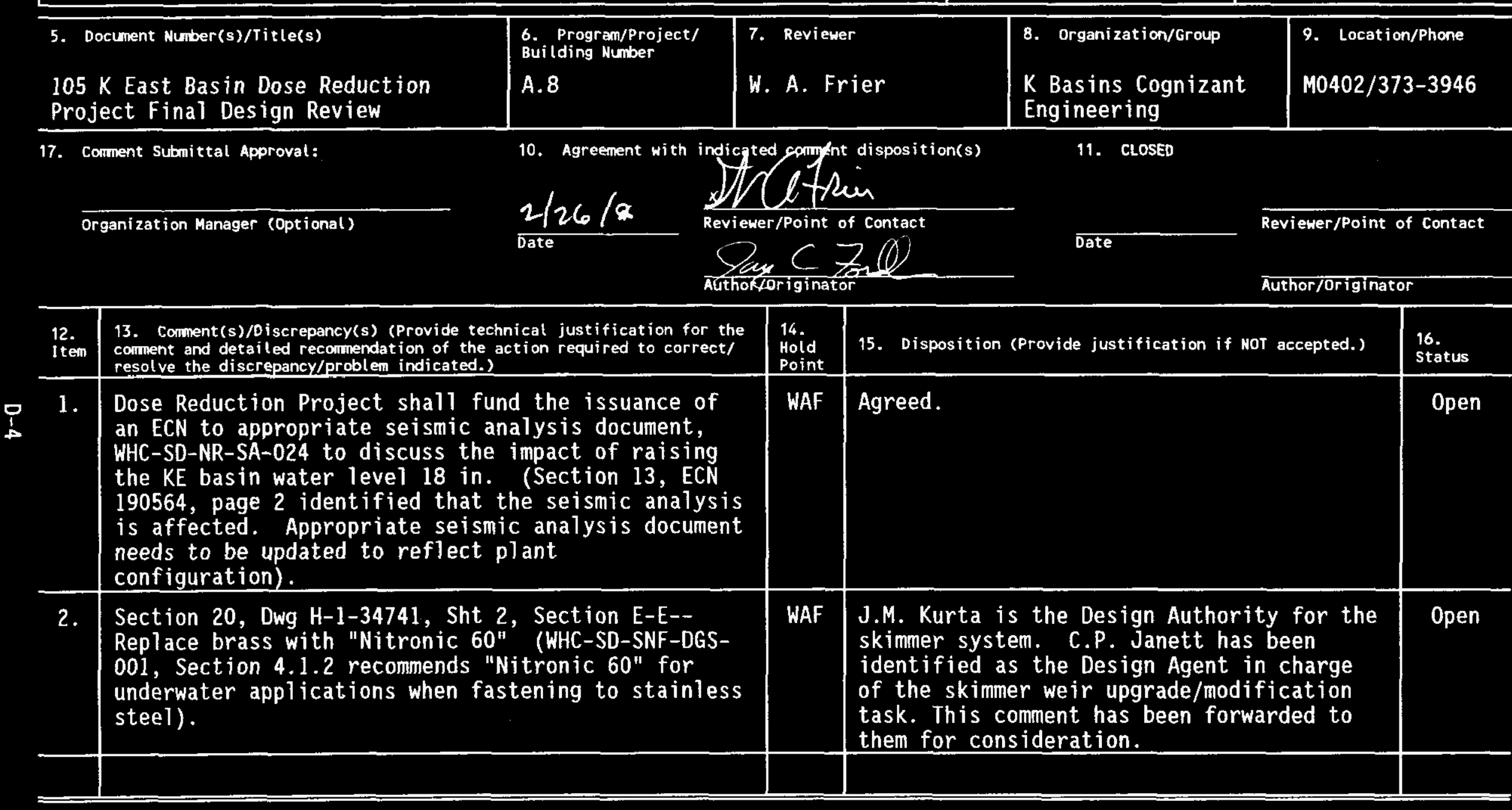




\begin{tabular}{|c|l|l|}
\hline \multirow{2}{*}{ REVIEW COMMENT RECORD (RCR) } & $\begin{array}{l}\text { 1. Date Review No. } \\
2 / 7 / 96\end{array}$ \\
\cline { 2 - 4 } & $\begin{array}{c}\text { 3. Project No. } \\
\text { A.8 Page }\end{array}$ & 2 of 2 \\
\hline
\end{tabular}

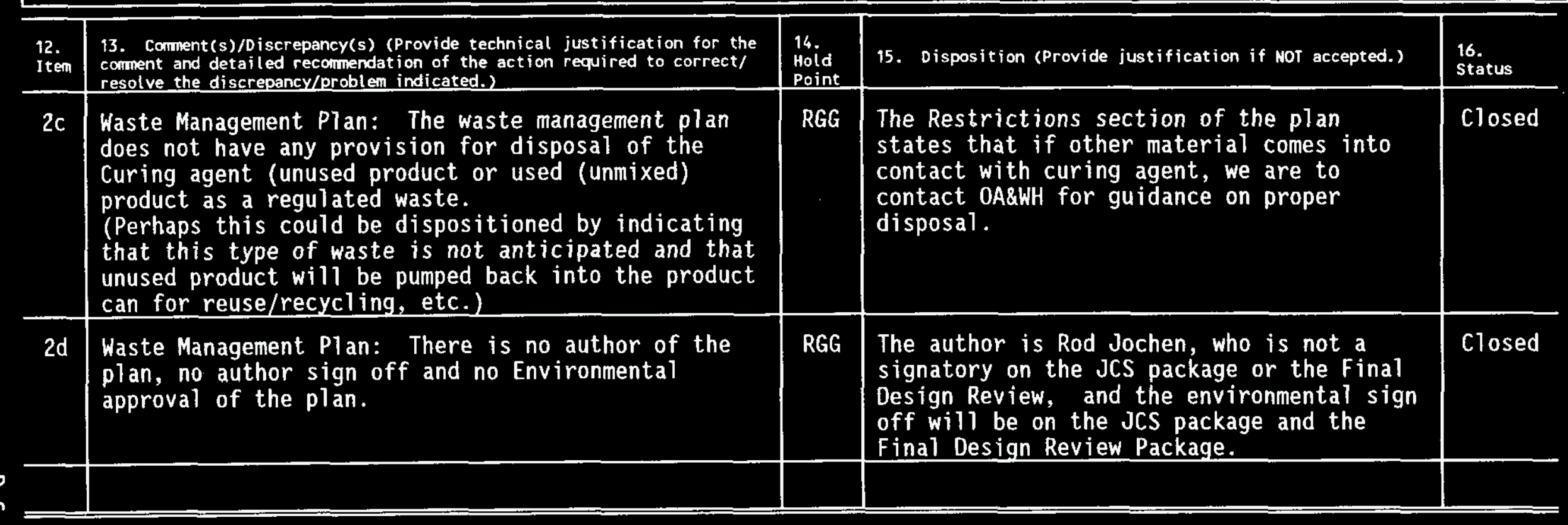


WHC-SD-SNF-DRR-006

Rev. 0

APPENDIX E

Action Item Data Base Status

E-1 
Project AIDB

\begin{tabular}{|c|c|c|c|c|c|c|c|c|c|c|}
\hline $\begin{array}{l}\text { ITEM } \\
\text { MUNBBER }\end{array}$ & ontew & DOCLMENT & COMBEENTER & DATE EMIERED & DESICN REVIEW COMMENT & ACTION/OASPOSMTION & RESPONGHELE & status & Dpen / closen. & TE COMOLETE \\
\hline \multicolumn{11}{|c|}{ 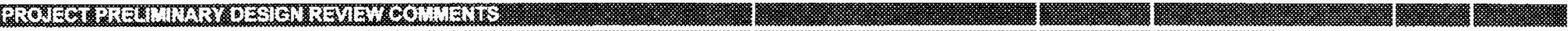 } \\
\hline 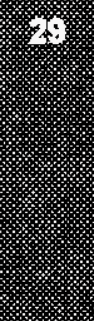 & is & 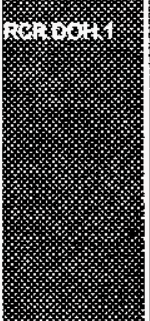 & 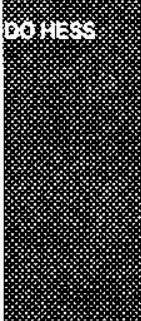 & 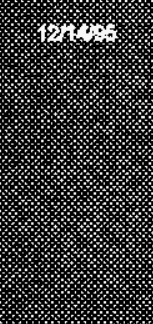 & 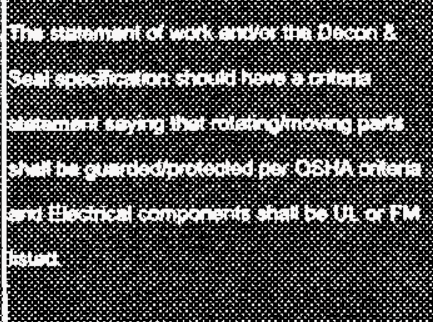 & 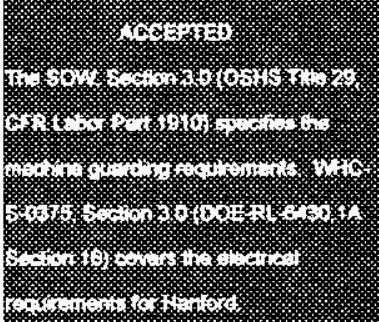 & m. & 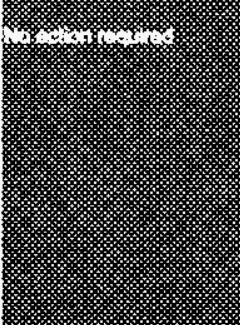 & 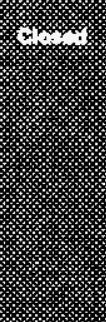 & 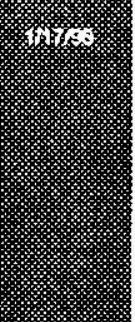 \\
\hline 30 & PROJ-PDR & RCR.DOH.2 & DOHESS & $12 / 14 / 95$ & $\begin{array}{l}\text { There is a potential criticality safety } \\
\text { issuehmat if relating to failure of the } \\
\text { equipment and dropping all or part of the } \\
\text { equipment into the basin on the stored fuel. }\end{array}$ & $\begin{array}{l}\text { ACCEPTED } \\
\text { This is covered by previous analyses } \\
\text { for fuelcanister dops and was } \\
\text { revisited for proposed core drilling } \\
\text { of the basin walls project. A } \\
\text { letter will be oblained from the } K \text {. } \\
\text { Basin Criticality Safety } \\
\text { Representative indicating that this } \\
\text { occidert is bounded by existing } \\
\text { documents. }\end{array}$ & Jones & & Open & \\
\hline 31 & PROS-POR & RCR.DOH.3 & DO HESS & $12 / 14 / 95$ & 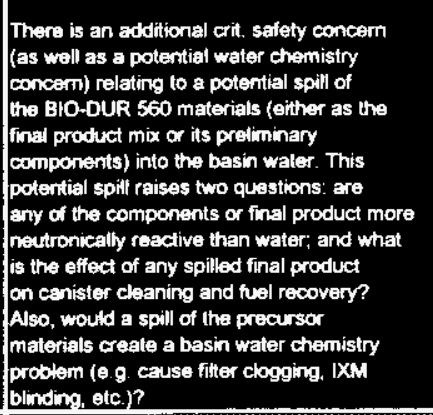 & $\begin{array}{l}\text { ACCEPTED } \\
\text { Steve Burke will evaluate the } \\
\text { coating material and document his } \\
\text { findings. The USQ screening and } \\
\text { Hazard Classification will evaluate } \\
\text { and document potential accident } \\
\text { scenarios, including the effects of } \\
\text { spillad coatiting malerial on the open } \\
\text { fuel and canisters. }\end{array}$ & Jones & & Open & \\
\hline 多 & 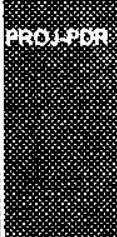 & 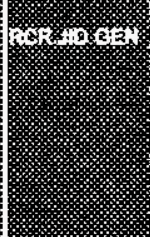 & (z) & $x_{k}^{\prime}$ & 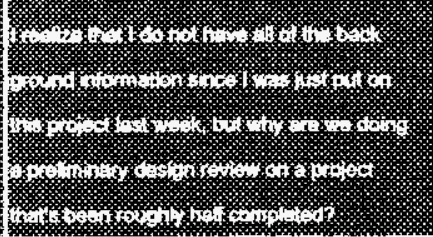 & r. & 淁淁: & 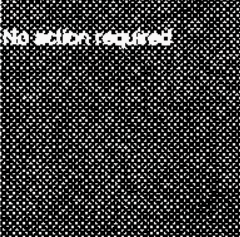 & 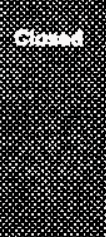 & 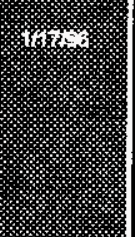 \\
\hline
\end{tabular}




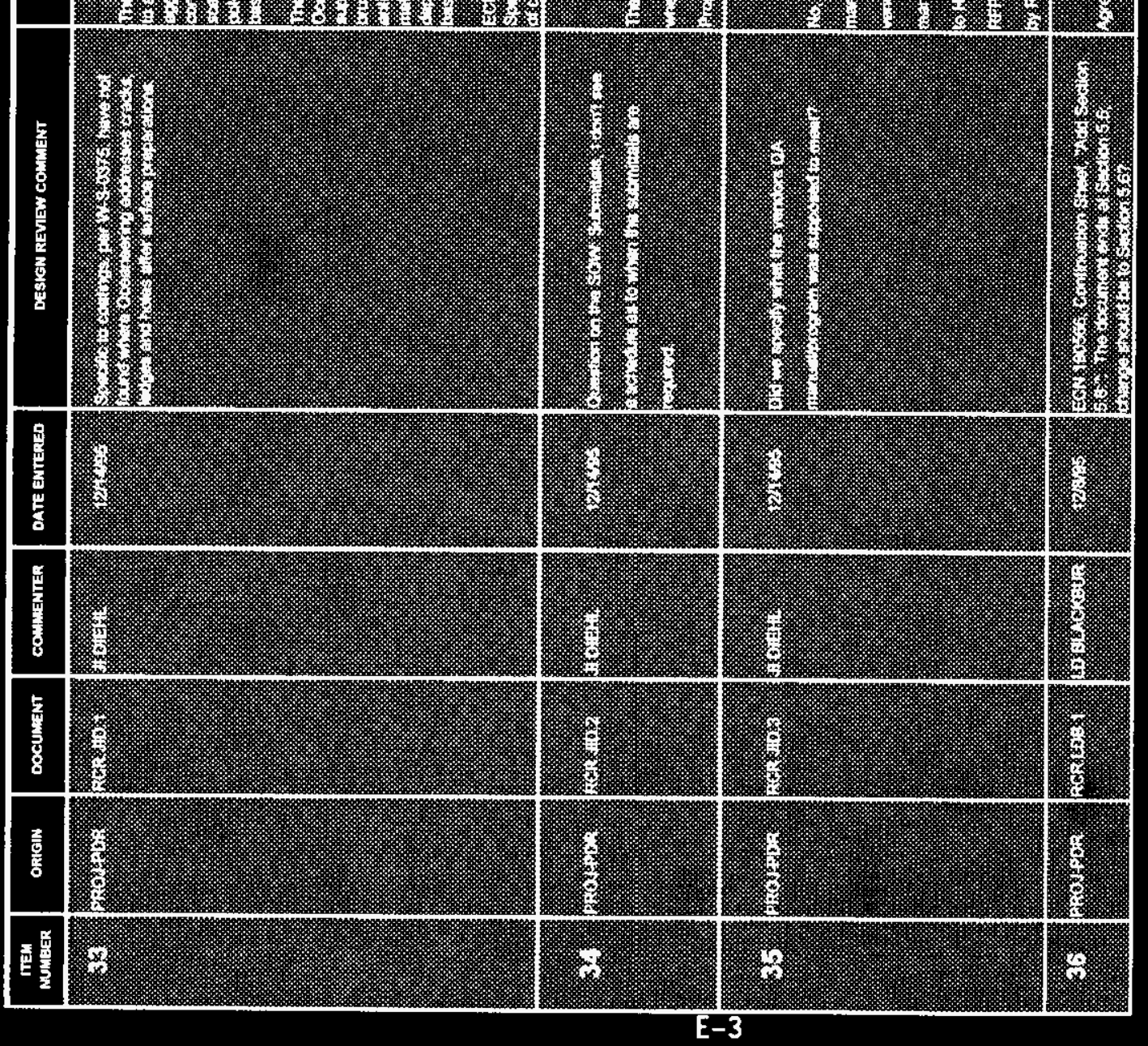




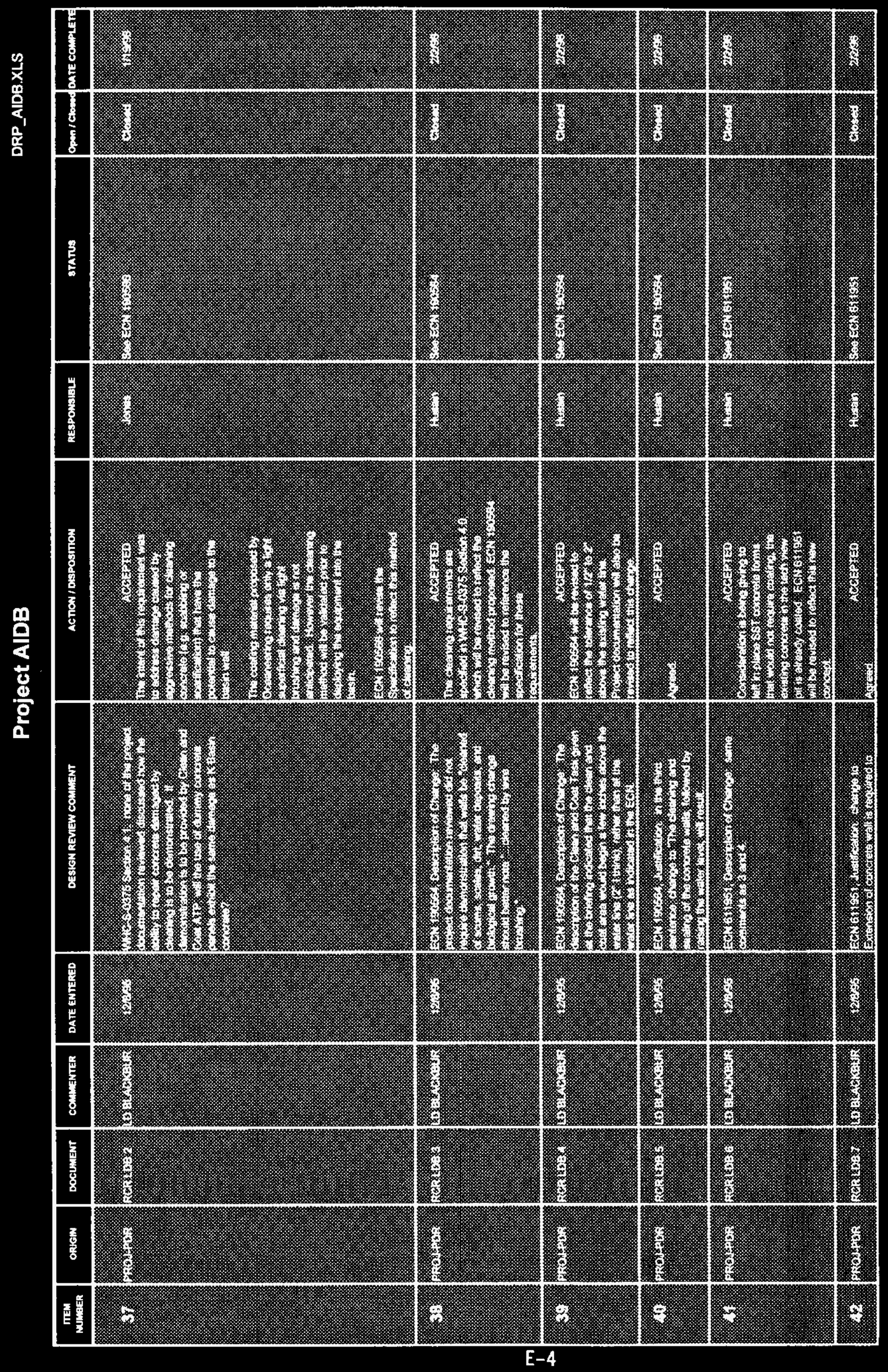




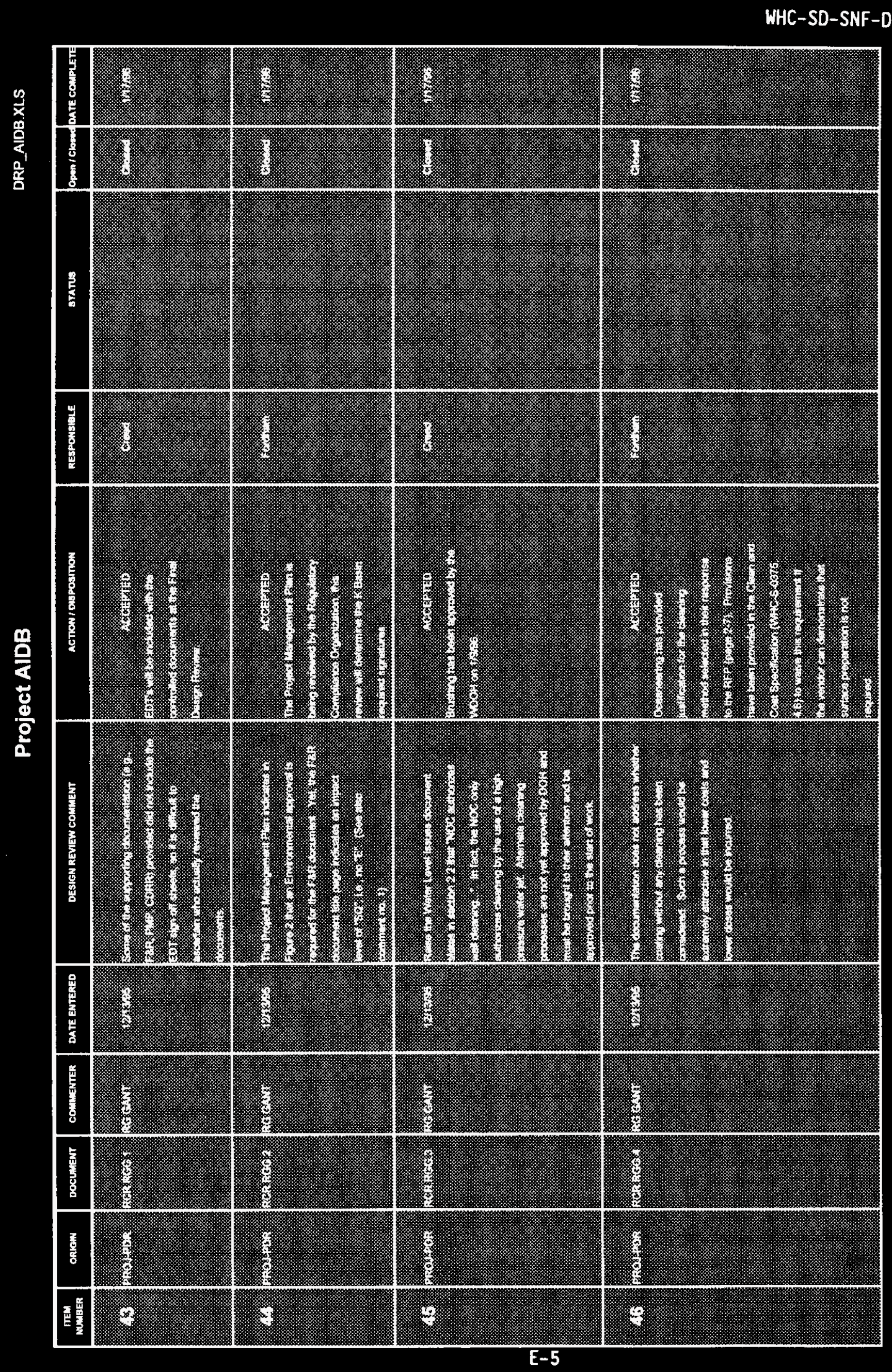




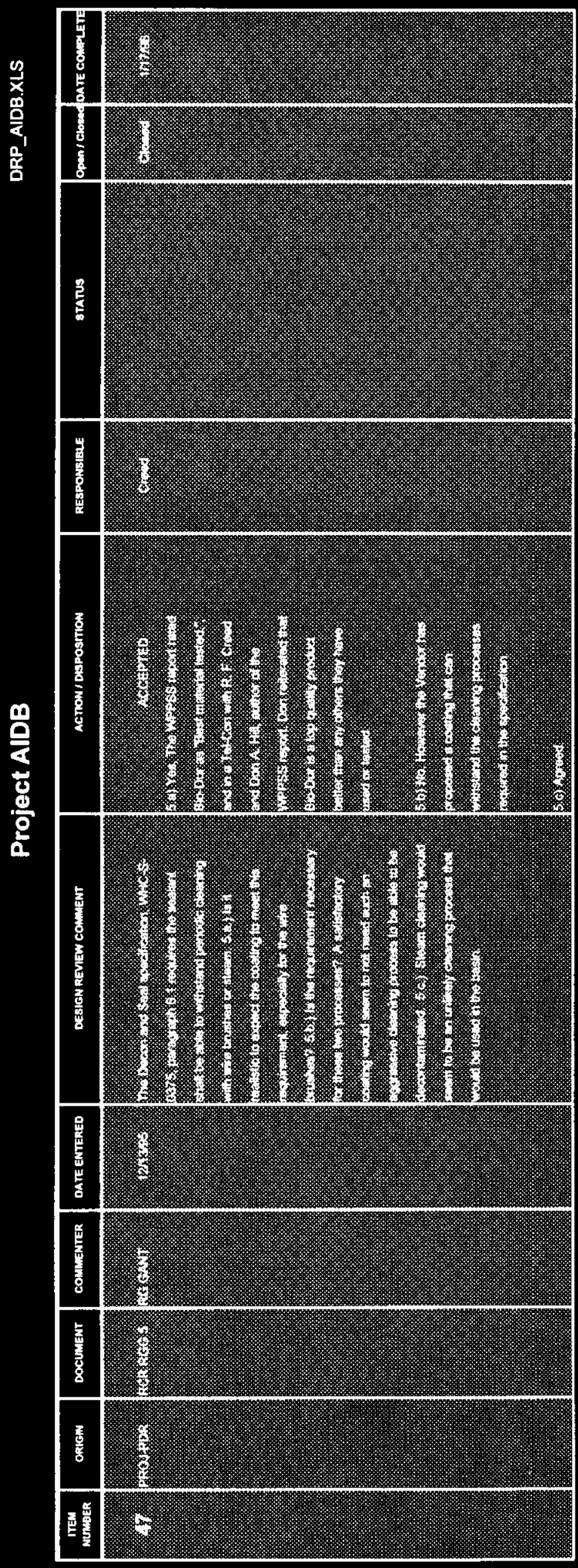

WHC-SD-SNF-DRR-006

Rev. 0

$\frac{9}{\stackrel{1}{*}}$

แ 


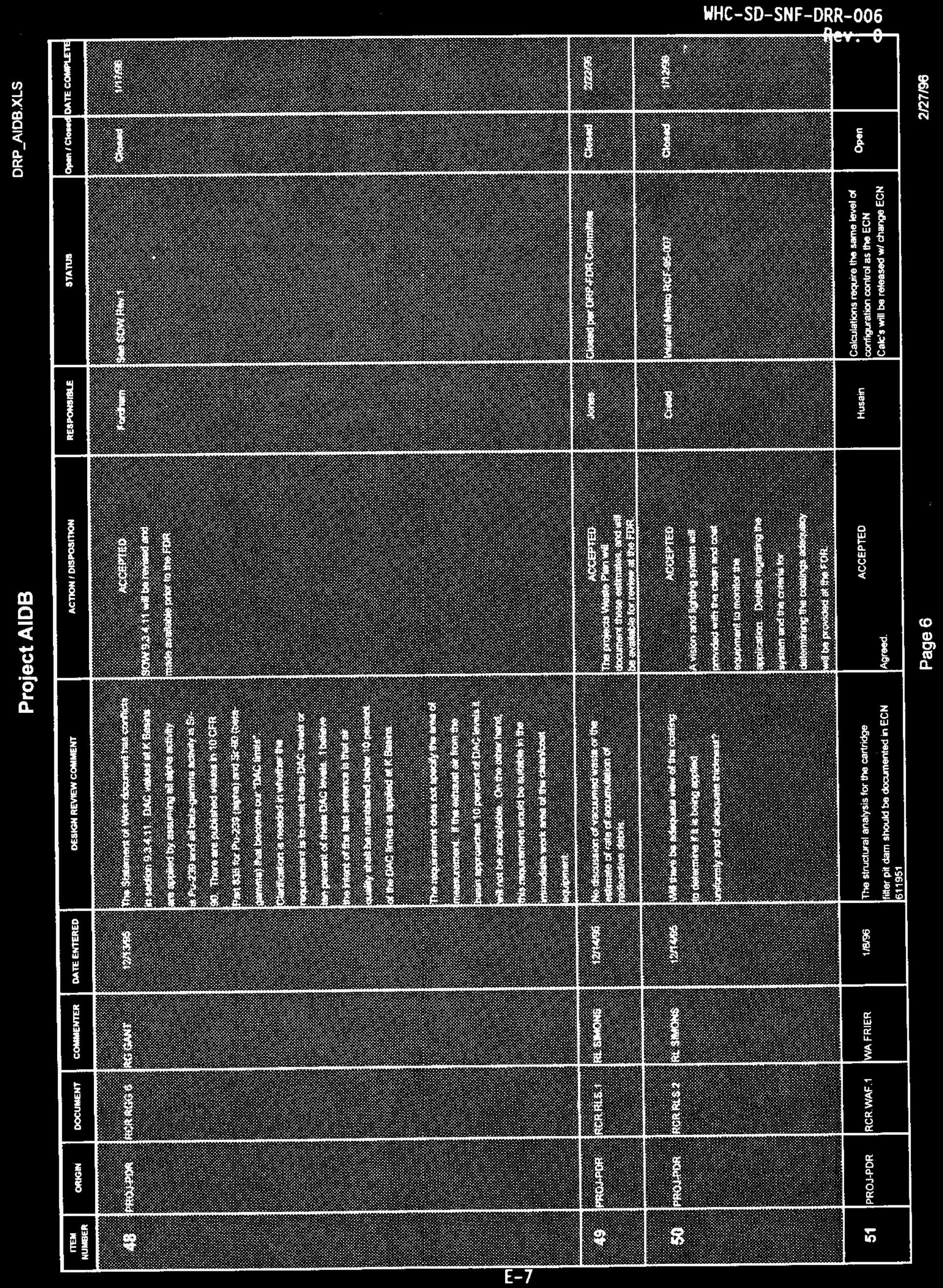




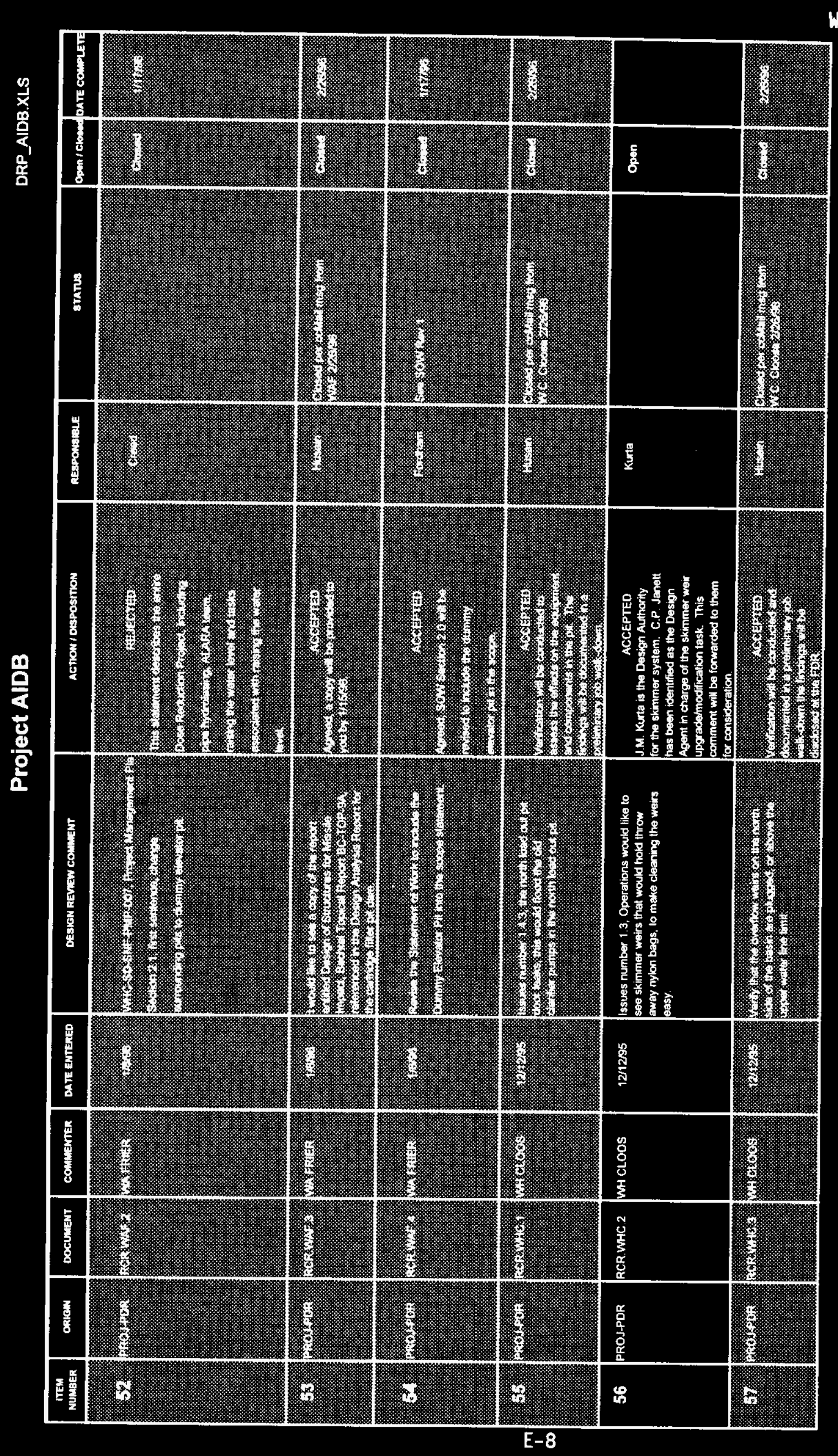

Rev. 0

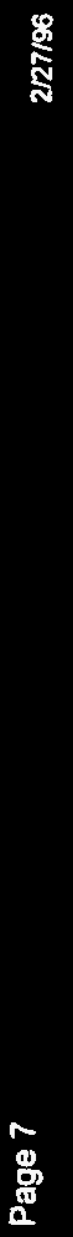




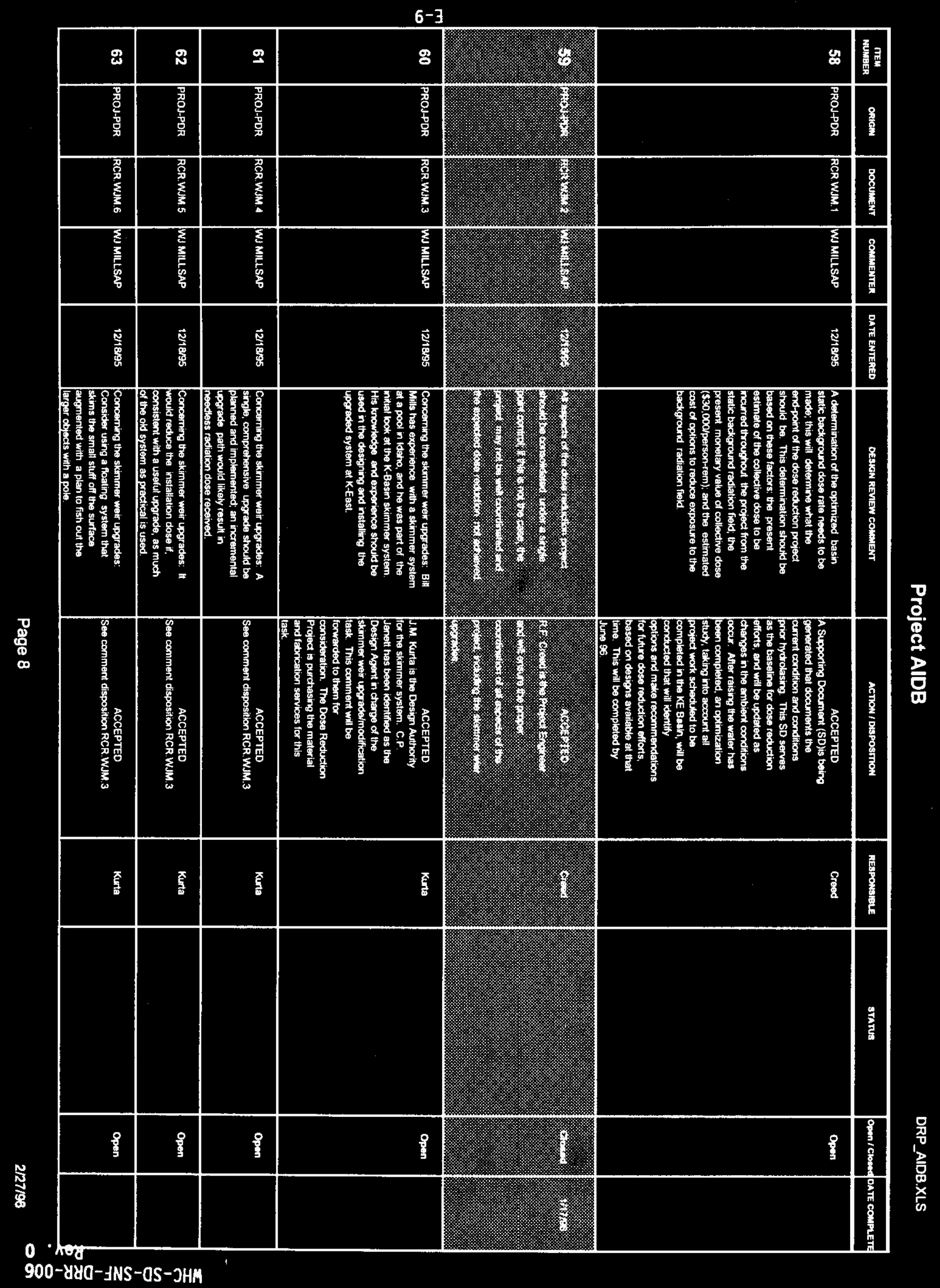


Project AIDB

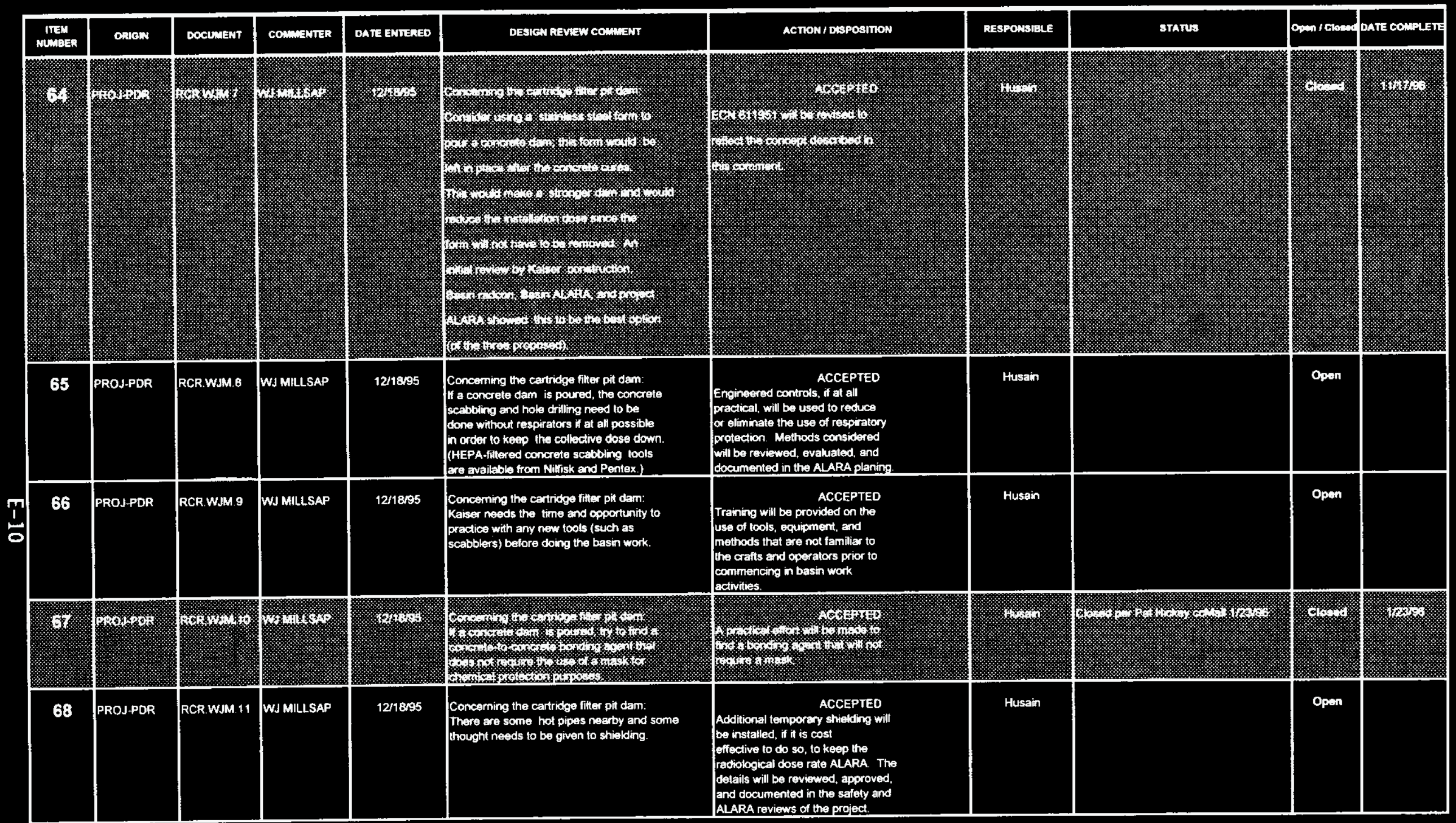




\section{APPENDIX $\mathbf{F}$}

\section{ACTION ITEN LIST}

1. Conduct a preliminary job walk-down to verify and assess a six-inch diameter pipe at the east end of the dummy elevator pit about one foot above the water level. The pipe is shown on drawing H-1-34683. Make modifications as required.

2. Provide an Engineering Change Notice to the seismic analys is document WHC-SD-NR-SA-024 to discuss the impact of raising the K-East Basin water level eighteen inches.

3. Forward to the design agent for the skimmer weir upgrade/modification the information that WHC-SD-SNF-DGS-001 recommends Nitronic 60 for underwater applications when fastening to stainless steel. This information suggests replacing brass with Nitronic 60 on Drawing H-1-34741, Sht. 2, Section E-E. 


\section{APPENDIX G}

\section{K BASIN DOSE REOUCTION PROJECT \\ FINAL DESIEN REVIEN}

\section{MEETING MINUTES}

The Final Design Review Meeting for the $\mathrm{K}$ Basin Dose Reduction Project was held on February 22, 1996 in the M0293 conference room at $2 \mathrm{p} . \mathrm{m}$. Those in attendance were:

*L. D. Blackburn, Chairman

*W. J. Millsap

*W. H. Cloos

*R. G. Gant

*J. I. Dieh1

R. F. Creed

J. C. Fordham

*D. 0 . Hess

*L. E. Formo

F. W. Moore

K. M. Jones

* Committee members

The agenda for the meeting was a review of all comments and dispositions documented on Review Comment Record (RCR) forms, review of the status of action items from the Preliminary Design Review, and completion of the design review checklist. The final RCRs resulting from meeting discussions and the completed design review checklist will be included in the design review report.

Discussions of the comments did not reveal any major items of concern. The conclusion of the committee was that the existing documents constitute an acceptable design for the Dose Reduction Project. 